\title{
S100A11 promotes human pancreatic cancer PANC-1 cell proliferation and is involved in the PI3K/AKT signaling pathway
}

\author{
MINGBING XIAO $^{1 *}$, TAO $^{2} I^{2 *}$, YIFEI JI ${ }^{1}$, FENG JIANG $^{1}$, WENKAI NI ${ }^{1}$, \\ JING ZHU $^{1}$, BAIJUN BAO ${ }^{1}$, CUIHUA LU ${ }^{1}$ and RUNZHOU NI ${ }^{1}$ \\ ${ }^{1}$ Department of Gastroenterology, Affiliated Hospital of Nantong University, Nantong, Jiangsu 226001; \\ ${ }^{2}$ Department of Medical Oncology, Nantong Tumor Hospital, Nantong, Jiangsu 226361, P.R. China
}

Received September 2, 2016; Accepted September 22, 2017

DOI: $10.3892 / \mathrm{ol} .2017 .7295$

\begin{abstract}
S100A11, a member of S100 calcium-binding protein family, is associated with the numerous processes of tumorigenesis and metastasis. In the present study, the role of S100A11, and its possible underlying mechanisms in cell proliferation, apoptosis and cell cycle distribution in human pancreatic cancer were explored. Immunohistochemical analyses of S100A11 and phosphorylated (p)-AKT serine/threonine kinase (AKT) were performed in 30 resected specimens from patients with pancreatic cancer. PANC-1 cells were transfected with pcDNA3.1-S100A11 or treated with $50 \mu \mathrm{mol} / 1 \mathrm{LY} 294002$ for $48 \mathrm{~h}$. Cell proliferation was determined using a cell counting kit-8 assay, whereas apoptosis and cell cycle distribution were determined by flow cytometry analysis. The mRNA and protein levels of S100A11, and AKT were determined using semi quantitative reverse transcription-polymerase chain reaction and western blot analyses, respectively. Pearson correlation analysis revealed that the expression levels of S100A11 and p-AKT were positively correlated $(r, 0.802 ; \mathrm{P}<0.05)$. Compared with the control group, S100A11 overexpression significantly promoted PANC-1 cell proliferation and reduced the percentage of early apoptotic cells. Flow cytometric analysis indicated that the proportion of PANC-1 cells in the S phase was significantly elevated and cell percentage in the G0/G1 phase declined in response to S100A11 overexpression (all $\mathrm{P}<0.05$ ). S100A11 overexpression also significantly increased AKT mRNA and p-AKT protein expression levels (both $\mathrm{P}<0.05$ ). The phosphoinositide 3-kinase (PI3K) inhibitor, LY294002, significantly inhibited PANC-1 cell proliferation, promoted apoptosis and caused G1/S phase arrest in PANC-1 cells (all $\mathrm{P}<0.05$ ). These
\end{abstract}

Correspondence to: Professor Runzhou Ni or Professor Cuihua Lu, Department of Gastroenterology, Affiliated Hospital of Nantong University, 20 Xisi Road, Nantong, Jiangsu 226001, P.R. China

E-mail: 79468048@qq.com

E-mail: 1ch670608@sina.com

*Contributed equally

Key words: S100A11, AKT serine/threonine kinase phosphorylation, pancreatic cancer, cell proliferation, apoptosis, cell cycle distribution findings together suggest that S100A11 promotes the viability and proliferation of human pancreatic cancer PANC-1 cells through the upregulation of the PI3K/AKT signaling pathway. Thus, S100A11 may be considered as a novel drug target for targeted therapy of pancreatic cancer.

\section{Introduction}

Pancreatic cancer is a highly malignant cancer type with a median survival of 3-6 months and a 5-year survival rate of $<5 \%$ worldwide (1-4). In 2015, compared with Western countries, the incidence of pancreatic cancer in China increased significantly. In 2010, 34,509 men and 23,226 women succumbed to pancreatic cancer in China (5). Furthermore, in 2015, 62.9\% of pancreatic cancer cases are diagnosed at stages I or II, and $37.1 \%$ of cases at stages III and IV (6). Chemotherapy is one of the main first-line treatments for advanced pancreatic cancer (7). However, controversy remains regarding its use due to its invasive nature (8). Hence, it is necessary to develop strategies, and identify novel potential pharmacological targets at the molecular level, which are involved in the signaling pathways associated with the occurrence, development and metastasis of pancreatic cancer.

S100A11 is a member of S100 calcium-binding protein family. Interaction of S100A11 with calcium leads to conformational changes of S100A11 protein resulting in the exposure of its hydrophobic region. This region in turn binds to the target protein to transduce the regulated signals of calcium-dependent cells. Thus, S100A11 protein is involved in multiple biological processes, including cell proliferation, apoptosis, signal transduction, cell adhesion, extracellular matrix remodeling and cell mobility (9-11). At present, it has been demonstrated that S100 protein is associated with the occurrence, development and metastasis of tumors (12). The S100 gene is located in the chromosome region Iq21. Tumor genes in this region are frequently recombined, which may easily cause loss-of-control of S100 gene expression (13). Furthermore, it has been demonstrated that the expression of S100A11 is upregulated in the early stage of pancreatic cancer and decreases with the progression of pancreatic cancer (13). S100A11 may serve as a significant tumor marker for pancreatic cancer and high expression of S100A11 is an unfavorable predictor for prognosis of patients with pancreatic cancer who have undergone surgical resection (14-16). 
The phosphoinositide 3-kinase (PI3K)/AKT serine/threonine kinase (AKT) pathway is involved in the growth, differentiation, and proliferation of somatic and cancer cells (17). Activation of the PI3K/AKT signaling pathway may result in increased tumor cell proliferation and the inhibition of tumor cell apoptosis $(17,18)$. As a key protein in the pathway, AKT serves an important role in regulating cell viability, growth and proliferation $(17,18)$. PI3K is an upstream effector of the PI3K/AKT signaling pathway, and may induce the activation of AKT through phosphorylating threonine and/or serine residues. PI3K inhibitor LY294002 is able to inhibit AKT phosphorylation (18). It has been reported that the PI3K/AKT signaling pathway is significantly active in pancreatic cancer cells (19). Additionally, immunohistochemical analysis demonstrated that the phosphorylated (p)-AKT positive rate was $46 \%$ in pancreatic cancer tissues, which was significantly higher compared with that in normal pancreatic tissues (17). Therefore, it has been considered that p-AKT is associated with poor prognosis of patients with pancreatic cancer (20). In vitro and in vivo experiments have suggested that the blockade of the PI3K/AKT signaling pathway significantly impairs the proliferation of pancreatic cancer cells, promotes its apoptosis and induces cell cycle arrest (21).

Furthermore, in normal human keratinocytes, AKT phosphorylation is inhibited through downregulation of S100A11 expression in the cells, which leads to a decrease in AKT activity, indicating that S100A11 is involved in AKT activation (22). Therefore, we hypothesized that S100A11 is associated with the PI3K/AKT signaling pathway in the occurrence and development of pancreatic cancer. Thus, the present study aimed to investigate the effects of S100A11 overexpression on cell proliferation, cell apoptosis and cell cycle distribution in pancreatic cancer cells, and to explore potential mechanisms associated with the PI3K/AKT signaling pathway.

\section{Materials and methods}

Patients and tissue specimens. Pancreatic paraffin samples were provided by the Department of Pathology, Affiliated Hospital of Nantong University (Nantong, China). There were 30 resection specimens from patients with pancreatic cancer hospitalized between January 2010 and June 2013 (male:female, 17:13; median age, 67 years; age range, 41-85 years. The incised margins were all $>1 \mathrm{~cm}$. Pathological diagnosis of all the cases was clear, and all the clinicopathological data were complete. None of the patients recruited in the present study had received radiotherapy or chemotherapy, or any other treatment prior to surgery. The Clinical Research Ethics Committee of the Affiliated Hospital of Nantong University approved the present study. All patients provided written informed consent for the use of their medical records and tissue specimens for research purposes.

Immunohistochemical analysis. The tissue sections were deparaffinized in xylene twice for $5 \mathrm{~min}$ at room temperature and then rehydrated using a graded ethanol series $(100,95$, 80 and $70 \% ; 5$ min at room temperature for each concentration). Subsequently, the endogenous peroxidase activity was blocked by soaking in $0.3 \%$ hydrogen peroxide for $10 \mathrm{~min}$ at room temperature. Thereafter, the sections were processed in
$10 \mathrm{mmol} / \mathrm{l}$ citrate buffer $\left(\mathrm{pH}\right.$ 6.0) and were heated to $121^{\circ} \mathrm{C}$ in an autoclave for $20 \mathrm{~min}$ to retrieve the antigen. After being rinsed with PBS (pH 7.2), 10\% goat serum (Seebio Biotechnology Co. Ltd, Shanghai, China) was added and incubated at room temperature for $1 \mathrm{~h}$ to block the non-specific reactions. The sections were then incubated overnight at $4^{\circ} \mathrm{C}$ with mouse anti-human S100A11 monoclonal antibody (cat. no. WH0006282M1; diluted 1:100; Sigma-Aldrich; Merck KGaA, Darmstadt, Germany) and rabbit anti-human p-Akt monoclonal antibody (cat. no. 4060; diluted 1:100; Cell Signaling Technology Inc., Danvers, MA, USA). Negative control slides were also processed in parallel using a non-specific IgG (cat. no. 18015; Sigma-Aldrich; Merck $\mathrm{KGaA}$ ) at the same concentration as the primary antibody. All slides were processed using the peroxidase antiperoxidase method (Dako; Agilent Technologies, Inc., Santa Clara, CA, USA). After being rinsed with PBS, the peroxidase reaction was visualized by incubating the sections with 3,3-diaminobenzidine tetrahydrochloride for $5 \mathrm{~min}$ at room temperature. The sections were then rinsed with water, counterstained with hematoxylin for $1 \mathrm{~min}$ at room temperature, then dehydrated and coverslipped.

Immunohistochemical evaluation. All of the immunostained sections were evaluated in a blinded manner by three independent experienced observers without any knowledge on the clinicopathological features of the patients. For assessment of S100A11 and p-AKT, five fields (magnification, $x 40$ ) in each specimen were selected randomly, and nuclear staining was examined using a light microscope. By counting the number of cells per field of view, the rate of cells expressing S100A11 and p-AKT was calculated; meanwhile, the staining intensity was observed. The positively stained areas were noted as those with light yellow to brown particles, and the specific expression in each case was defined using the staining intensity score and percentage of positively stained cells. The staining intensity scoring (according to the degree of color) was as follows: 0 , no staining; 1, pale yellow diffused distribution from cytoplasm to cytoplasmic membrane; 2 , brown distribution with fine granular; 3 , brown or dark brown distribution with coarse granular from cytoplasm to cytoplasmic membrane as well as nucleus. The percentage of positively stained cells was categorized as follows: $0,<5 \% ; 1,5-25 \% ; 2,26-50 \% ; 3,51-75 \% ; 4$, $>75 \%$. The score of each patient was calculated as the staining intensity score x positively stained cells score. In $50 \%$ of the samples, staining was repeated twice to avoid technical errors, but similar results were obtained in these samples.

Cell culture. The human pancreatic cancer PANC-1, Bxpc-1 and AsPC-1 cell lines were purchased from the Type Culture Collection of the Chinese Academy of Sciences (Shanghai, China). Cells were maintained in RPMI-1,640 supplemented with $10 \%$ heat-inactivated fetal calf serum, $2 \mathrm{mM} \mathrm{L}$-glutamine, and $100 \mathrm{U} / \mathrm{ml}$ penicillin and streptomycin mixture (all from Gibco, Thermo Fisher Scientific, Inc., Waltham, MA, USA) at $37^{\circ} \mathrm{C}$ with $5 \% \mathrm{CO}_{2}$.

S100A11 overexpression. RNA was extracted from PANC-1 using the RNeasy Mini kit (Qiagen, Inc., Valencia, CA, USA), and cDNA was synthesized via reverse transcription (RT) 
with the Sensiscript RT kit (Qiagen, Inc.) according to the manufacturer's protocol. The cDNA was used as the template to amplify the S100A11 gene using the S100A11 primers as follows: Forward, 5'-CTCGGATCCGCCACCATGGCAAAA ATCTCCAGCCCTACAG-3' (BamH I) and reverse, 5'-GCC GCTCGAGGGGTCCTCAGGTCCGCTTCTG-3' (Xhol I). The control gene ( $\beta$-actin) primers were as follows: Forward, 5'-AAGTACTCCGTGTGGATCGG-3' and reverse, 5'-ATG CTATCACCTCCCCTGTG-3'. The polymerase chain reaction (PCR) theremocycling parameters were as follows: $95^{\circ} \mathrm{C}$ for $30 \mathrm{sec}$ followed by 35 cycles of $94^{\circ} \mathrm{C}$ for $45 \mathrm{sec}$ and $55^{\circ} \mathrm{C}$ for $45 \mathrm{sec}$. The PCR product was run using agarose gel electrophoresis, and was then extracted and purified. The purified DNA and pcDNA3.1/myc-HisA (eukaryotic expression vector, Invitrogen; Thermo Fisher Scientific, Inc.) were digested using the restriction enzymes BamH I-HF, Xhol I (both from New England BioLabs, Inc., Ipswich, MA, USA). The digested insert (PCR product) and vector (pcDNA3.1/myc-HisA) were ligated using T4 DNA ligase (New England BioLabs, Inc.). Subsequently, the ligation product was transformed into E.coli competent cells and the competent cells were plated onto Luria-Bertani broth (LB) plates. On the second day, the white colonies were picked from the LB plates, followed by plasmid extraction and sequencing. Finally, the plasmid was named as pcDNA3.1-S100A11. Lipofectamine 2000 (Invitrogen; Thermo Fisher Scientific, Inc.) was used to transfect PANC-1 cells with $2.5 \mu \mathrm{g}$ pcDNA3.1-S100A11 and pcDNA3.1 empty vector.

Drug application. PANC-1 cells were seeded into 96-well plates $\left(5 \times 10^{3}\right.$ cells/well). After $24 \mathrm{~h}$, the medium was removed and replaced with LY294002 (Beyotime Institute of Biotechnology, Haimen, China) dissolved in $50 \mu \mathrm{mol} / 1$ dimethylsulfoxide (DMSO) according to a previous protocol (19). The concentration of LY294002 used in experiments was $50 \mu \mathrm{mol} / 1$, and was incubated with cells for $48 \mathrm{~h}$ at $37^{\circ} \mathrm{C}$.

Cell proliferation assay. Cell proliferation was measured by a commercial Cell Counting kit (CCK)-8 (Dojindo Molecular Technologies, Inc., Kumamoto, Japan) following the manufacturer's protocol. Briefly, PANC-1 cells were seeded onto 96-well cell culture plates at a concentration of $2 \times 10^{4}$ cells/well in $100 \mu \mathrm{l}$ culture medium as aforementioned, and were grown overnight. CCK-8 reagent $(10 \mu \mathrm{l})$ was added to a subset of wells and incubated for $2 \mathrm{~h}$ at $37^{\circ} \mathrm{C}$ and the absorbance reading was taken using an automated plate reader under the detection wavelength of $450 \mathrm{~nm}$.

Cell cycle distribution determined by flow cytometric analysis. For cell cycle analysis, cells were harvested, washed once in PBS, fixed with $70 \%$ ethanol at $-20^{\circ} \mathrm{C}$ overnight and then incubated with $1 \mathrm{mg} / \mathrm{ml}$ RNase A for $30 \mathrm{~min}$ at $37^{\circ} \mathrm{C}$. Subsequently, the cells were stained with propidium iodide (PI; $50 \mu \mathrm{g} / \mathrm{ml}$; BD Biosciences) in PBS-Triton X100 for an additional $20 \mathrm{~min}$ at $4^{\circ} \mathrm{C}$, and analyzed using the BD FACScan flow cytometer with CellQuest acquisition and analysis programs (FlowJo 10; FlowJo LLC, Ashland, OR, USA). Finally, the proportions of cells in the G0/G1, S and G2/M phases were determined.

Cell apoptosis determined by flow cytometric analysis. PANC-1 cell apoptosis was detected using an Annexin
V-FITC/PI staining assay. Flow cytometry analysis of apoptotic cells was performed using an Annexin V-FITC/PI staining kit (BD Biosciences). After washing with cold PBS, the cells were resuspended in binding buffer (100 mM HEPES, $\mathrm{pH} 7.4,100 \mathrm{mM} \mathrm{NaCl}$, and $25 \mathrm{mM} \mathrm{CaCl}_{2}$ ) followed by staining with Annexin V-FITC/PI at room temperature in the dark for $15 \mathrm{~min}$. Apoptotic cells were then evaluated by gating PI and Annexin V-positive cells on a FACS Calibur (BD Biosciences). Annexin V was set as the horizontal axis and PI was set for the vertical axis. Early apoptotic cells were located in the lower right quadrant of the flow cytometric dot plot.

Semi-quantitative RT-PCR. Total RNA from PANC-1 cells was extracted using a TRIzol extraction kit (Invitrogen; Thermo Fisher Scientific, Inc.) according to the manufacturer's protocol. Total RNA was reverse transcribed using the ThermoScript RT-PCR system (Invitrogen; Thermo Fisher Scientific, Inc.). The primer sequences were as follows: S100A11 forward, 5'-GAGTCCCTGATTGCTGTCTTC-3' and reverse, 5'-AGGGTCCTTCTGGTTCTTTG-3'; AKT forward, 5'-GGTATTTTGATGAGGAGTTCACG-3' and reverse, 5'-GAGTAGGAGAACTGGGGGAAGT-3'; $\beta$-actin forward, 5'-AAGTACTCCGTGTGGATCGG-3' and reverse, 5'-ATGCTATCACCTCCCCTGTG-3'. Thermocycling conditions were as follows: $94^{\circ} \mathrm{C}$ for $45 \mathrm{sec}, 55^{\circ} \mathrm{C}$ for $45 \mathrm{sec}, 72^{\circ} \mathrm{C}$ for $30 \mathrm{sec}$ for a total of 30 cycles. After amplification, the products were separated on a $1.5 \%$ agarose gel (cast in the presence of ethidium bromide) and the intensity of the bands was analyzed with Quantity One software (version 4.62; Bio-Rad Laboratories, Inc., Hercules, CA, USA).

Western blot analysis. PANC-1 cells were lysed in the lysis buffer (1 M Tris- $\mathrm{HCl}$ at $\mathrm{pH} 7.5,1 \%$ Triton X-100, 1\% Nonidet P-40, $10 \%$ SDS, $0.5 \%$ sodium deoxycholate, $0.5 \mathrm{M}$ EDTA, $10 \mu \mathrm{g} / \mathrm{ml}$ leupeptin, $10 \mu \mathrm{g} / \mathrm{ml}$ aprotinin, and $1 \mathrm{mM}$ phenylmethylsulfonyl fluoride) and then centrifuged at $4{ }^{\circ} \mathrm{C}$, $10,000 \mathrm{x} g$ for $30 \mathrm{~min}$ to collect the supernatant. Protein concentration was determined using the Bio-Rad protein assay kit (Bio-Rad Laboratories, Inc.). Equal amounts (0.6 mg/lane) of protein were separated with $10 \%$ gel using SDS-PAGE and transferred to polyvinylidine difluoride filter membranes (EMD Millipore, Billerica, MA, USA). Membranes were then blocked with $5 \%$ non-fat milk in TBS with $0.05 \%$ Tween 20 for $2 \mathrm{~h}$ at room temperature. Subsequently, the membrane was incubated for $12 \mathrm{~h}$ at $4^{\circ} \mathrm{C}$ with primary antibodies, including anti-S100A11 (1:500; cat. no. WH0006282M1; Sigma-Aldrich; Merck KGaA), anti-p-AKT (1:500; cat. no. 4060; Cell Signaling Technology, Inc.), anti-AKT (1:1,000; cat. no. 4685; Cell Signaling Technology, Inc.) and anti- $\beta$-actin (1:500; Santa Cruz Biotechnology Inc., Dallas, TX, USA). Next, membranes were incubated with horseradish peroxidase-conjugated IgG (1:4,000; anti-mouse IgG, cat. no. 7076; anti-rabbit IgG, cat. no. 7074; Santa Cruz Biotechnology, Inc.) for $1 \mathrm{~h}$ at room temperature. Immunoblotting bands were visualized by chemiluminescence (NEN Life Science Products, Inc., Boston, MA, USA). The band density was measured using a computer-assisted image-analysis system (Image Lab Software 5.2.x; Bio-Rad Laboratories, Inc.) and was normalized against the $\beta$-actin level. 

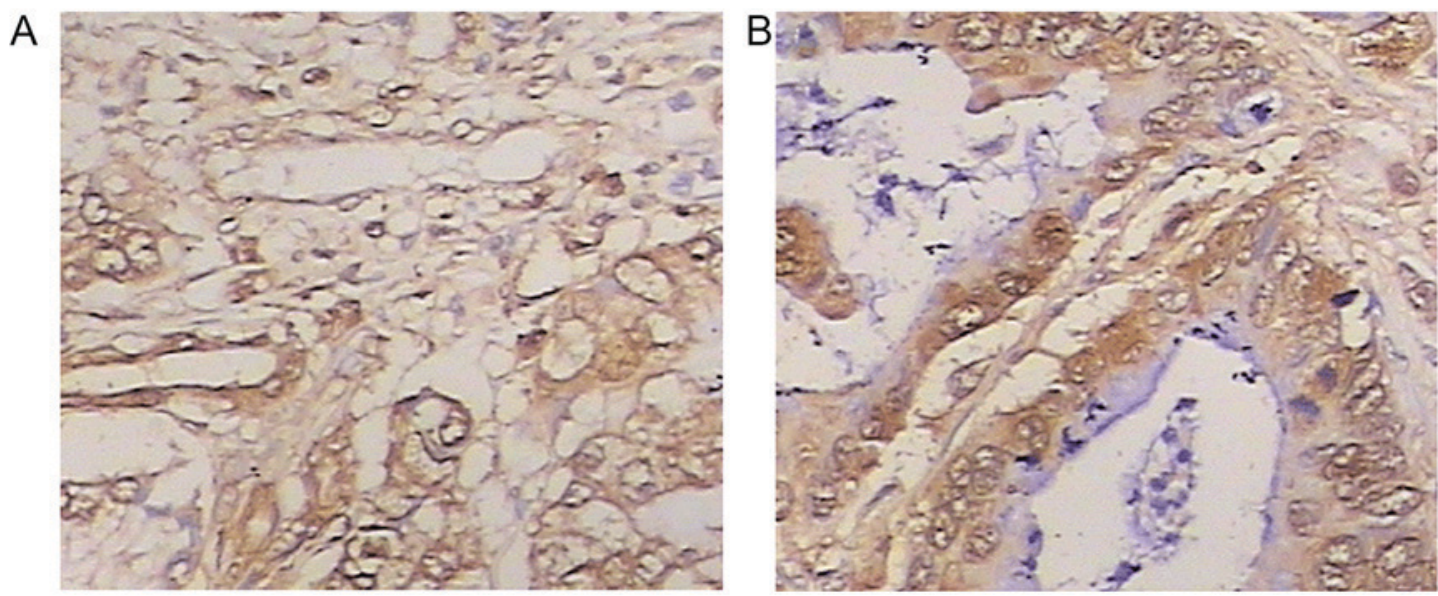

C

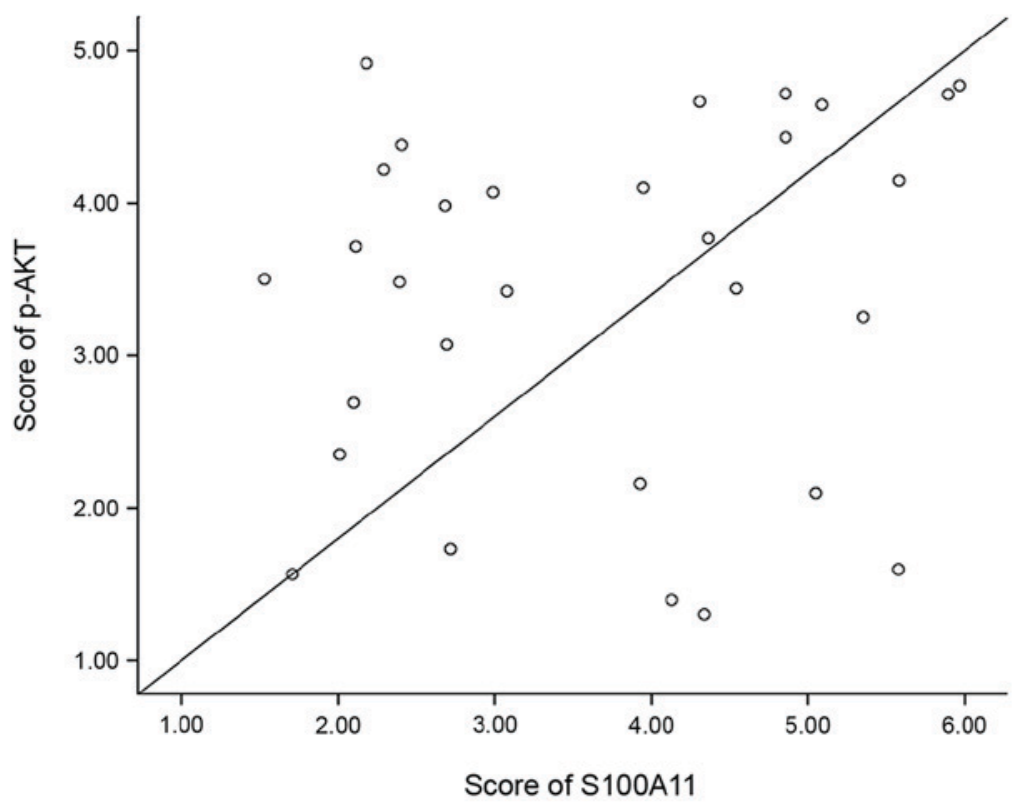

Figure 1. Correlative analysis of the expression levels of S100A11 and p-AKT in pancreatic cancer tissues. Immunohistochemical detection of the expression of (A) S100A11 and (B) p-AKT in pancreatic cancer tissues (magnification, x400). (C) Pearson correlation analysis revealing that in the 30 cases of pancreatic cancer tissue, the expression levels of S100A11 and p-AKT were positively correlated ( $r, 0.802 ; \mathrm{P}<0.05)$. p, phosphorylated; AKT, AKT serine/threonine kinase.

Statistical analysis. All analyses were performed using SPSS 19.0 software (IBM, Corp., Armonk, NY, USA). Data are presented as the mean \pm standard error of mean of at least three independent experiments. For statistical analysis, data analysis was performed using a Student's t-test or one-way analysis of variance with the Student-Newman-Keuls test. The expression levels of S100A11 and p-AKT in the pancreatic tissues were analyzed using Pearson's correlation analysis. $\mathrm{P}<0.05$ was considered to indicate a statistically significant difference.

\section{Results}

Correlation between S100A11 and p-AKT expression in human pancreatic paraffin tissue samples. Immunohistochemical analysis revealed that S100A11 was highly expressed in the cytoplasm and to a less extent in the nucleus, while p-AKT was expressed primarily in the cytoplasm with brown yellow or brown staining (Fig. 1A and B). Pearson correlation analysis revealed that the levels of S100A11 and p-AKT were positively

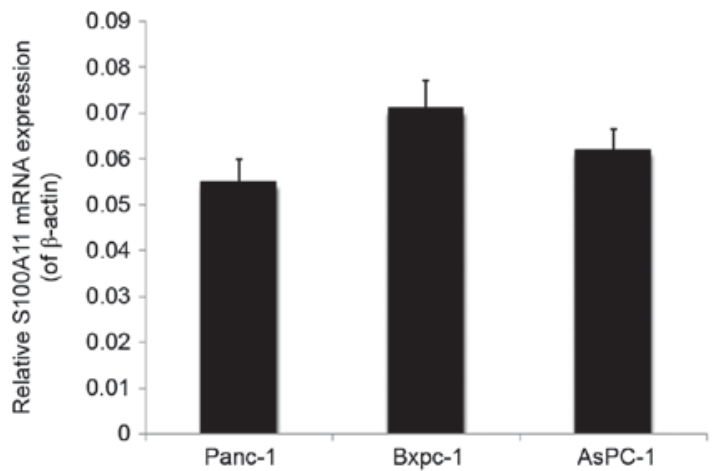

Figure 2. Semi-quantitative reverse transcription-polymerase chain reaction analysis of S100A11 mRNA expression in different pancreatic cancer cell lines relative to $\beta$-actin.

correlated (r, 0.802; P<0.05; Fig. 1C) among the 30 cases of pancreatic tissues. 
A

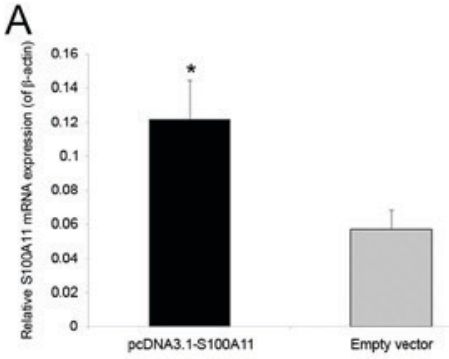

D
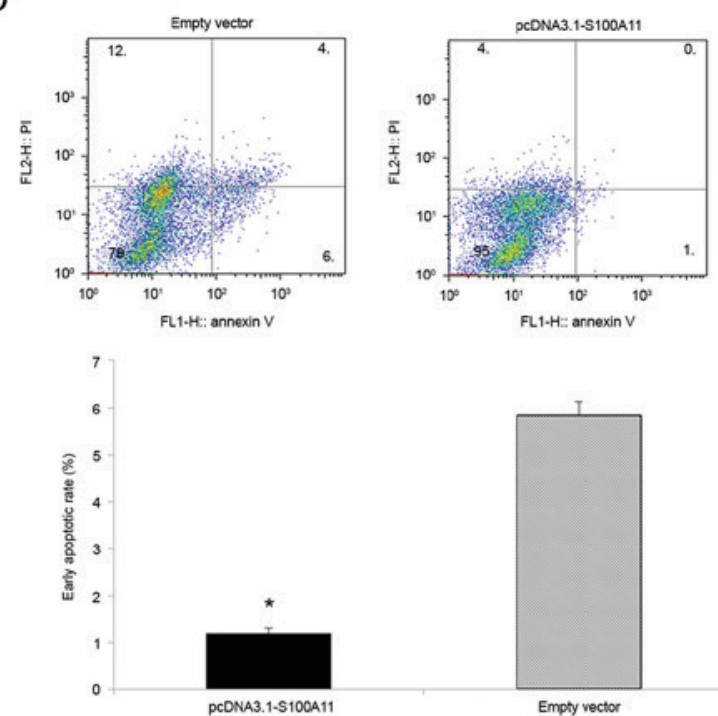

B

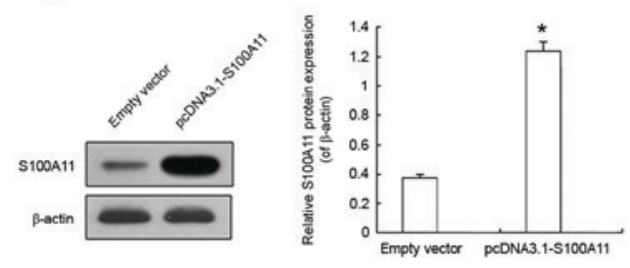

E

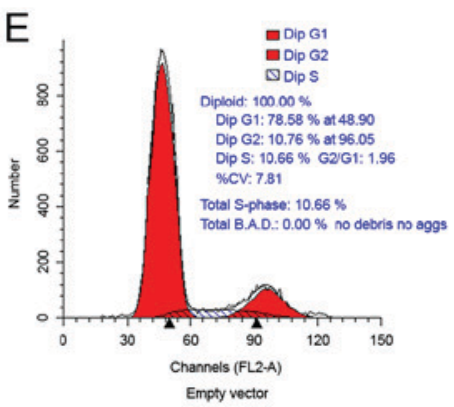

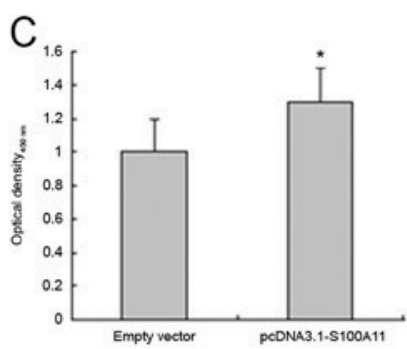

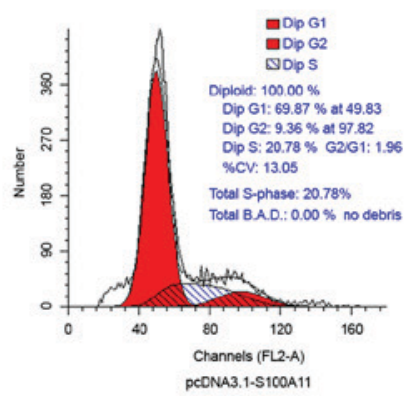

Figure 3. Effects of S100A11 overexpression on cell proliferation, cell apoptosis and cell cycle distribution in human pancreatic cancer PANC-1 cells. PANC-1 cells were transfected with 2.5 $\mu \mathrm{g}$ pcDNA3.1-S100A11 and pcDNA3.1 emptor vector using Lipofectamine 2000 for $48 \mathrm{~h}$. (A) S100A11 mRNA expression was determined by semi-quantitative reverse transcription-polymerase chain reaction analysis. $\beta$-actin was used as a control. (B) S100A11 protein expression was determined by western blot analysis. $\beta$-actin was used as a control. (C) Cell proliferation was determined using a cell counting kit- 8 assay. (D) Cell apoptosis was determined by flow cytometry analysis using Annexin V/PI staining. (E) Cell cycle distribution was determined by flow cytometry analysis using PI staining. Data were presented as the mean \pm standard error of mean following three independent experiments. ${ }^{*} \mathrm{P}<0.05$ vs. empty vector. PI, propidium iodide.

Effects of S100A11 overexpression on cell proliferation, cell apoptosis and cell cycle distribution in PANC-1 cells. To examine the role of S100A11 on the proliferation, apoptosis and cell cycle regulation of pancreatic cancer cells, semi-quantitative RT-PCR was used to analyze the expression profile of S100A11 in different pancreatic cancer cell lines including PANC-1, Bxpc-1 and AsPC-1 cells. The results demonstrated that PANC-1 had a relative low expression of endogenous S100A11 among these cell lines (Fig. 2). Accordingly, PANC-1 cell line was chosen for subsequent S100A11 overexpression experiments. Compared with the control group, the expression of S100A11 mRNA and protein in the cells transfected with pcDNA3.1-S100A11 was significantly increased (Fig. 3A and B). Through the CCK-8 experiment, the optical density was measured. Compared with cells transfected with the pcDNA3.1 empty vector, transfection with $2.5 \mu \mathrm{g}$ pcDNA3.1-S100A11 significantly promoted the proliferation of PANC-1 cells $(\mathrm{P}<0.05$; Fig. 3C). Furthermore, flow cytometry analysis was used to determine the level of apoptotic cell death of control and S100A11-overexpressing PANC-1 cells. The early apoptotic rate (\%) in the lower right quadrant of the flow cytometric dot plot in the cells transfected with pcDNA3.1-S100A11 was significantly reduced $(1.18 \pm 0.12$ vs. $5.83 \pm 0.30 \%$ in the control; $\mathrm{P}<0.05$; Fig. 3D). To further explore the involvement of S100A11 in the proliferation of pancreatic cancer cells, the cell cycle distribution of control and S100A11-overexpressing PANC-1 cells was detected (Fig. 3E). The proportion of cells in G0/G1 phase in pcDNA3.1-S100A11 and pcDNA3.1 groups was $68.81 \pm 5.42$, and $78.68 \pm 6.21 \%$, respectively $(\mathrm{P}<0.05)$, while the proportion of cells in the $\mathrm{S}$ phase was $20.48 \pm 2.37$ and $10.81 \pm 2.65 \%$, respectively $(\mathrm{P}<0.05)$.

Effects of S100A11 overexpression on AKT mRNA expression, $p-A K T$ and total AKT protein expression in PANC-1 cells. To further investigate the association between S100A11 and AKT, the mRNA and protein expression levels of AKT were analyzed. Compared with the empty vector group, AKT mRNA expression was significantly higher in the pcDNA3.1-S100A11 group (Fig. 4A; $\mathrm{P}<0.05$ ). In addition, the protein levels of $\mathrm{p}-\mathrm{AKT}$ and total AKT were significantly higher in the S100A11 overexpression group (Fig. 4B; $\mathrm{P}<0.05$ ).

Effects of PI3K inhibitor LY294002. LY294002 significantly inhibited the AKT mRNA expression and p-AKT protein expression when compared with the corresponding negative controls (Fig. 5A and B; $\mathrm{P}<0.05$ ). However, no significant differences were noted in the mRNA of S100A11, and protein expression levels of S100A11 and total AKT (Fig. 5A and B). PANC-1 cells were treated with $50 \mu \mathrm{mol} / 1$ of LY294002 for $12,24,48$ and $72 \mathrm{~h}$, followed by CCK-8 analysis. It 


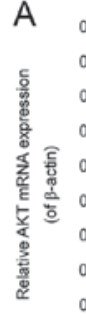

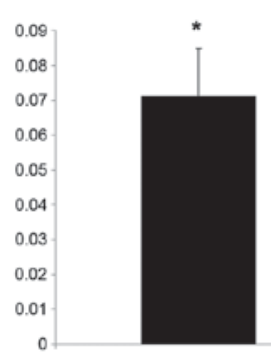

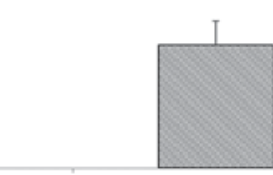

B

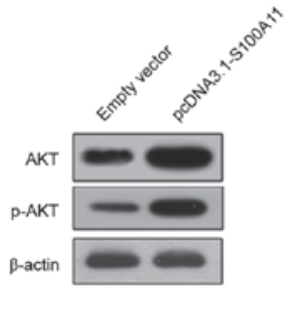

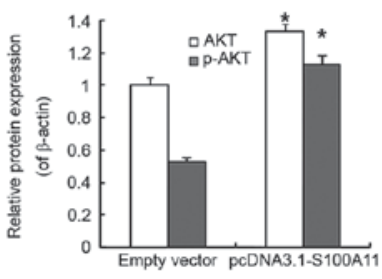

Figure 4. Effects of S100A11 overexpression on AKT mRNA expression, and p-AKT and total AKT protein expression in PANC-1 cells. (A) AKT mRNA expression was determined by semi-quantitative reverse transcription-polymerase chain reaction analysis. $\beta$-actin was used as a control. (B) Total AKT and p-AKT protein expression was determined by western blot analysis. $\beta$-actin was used as a control. Data were presented as the mean \pm standard error of mean following three independent experiments. ${ }^{*} \mathrm{P}<0.05$ vs. empty vector. p, phosphorylated; AKT, AKT serine/threonine kinase.

was demonstrated that LY294002 treatment resulted in a dose-dependent decrease in cell viability from $86.2 \%$ at $12 \mathrm{~h}$ to $37.4 \%$ at $72 \mathrm{~h}$ (Fig. 5C). As LY294002 treatment inhibited cell viability, the effect of LY294002 on PANC-1 cell apoptosis and cell cycle distribution was further examined. The proportion of early apoptotic cells was $21.36 \pm 2.34 \%$ in the lower right quadrant of the scatter plot in the LY294002 group, which was significantly higher compared with the proportion of early apoptotic cells in the control group $(1.18 \pm 0.12 \%$; $\mathrm{P}<0$.05; Fig. 5D). Compared with the control group, LY294002 treatment led to an increase of cells in the G0/G1 phase from $70.62 \pm 4.21$ to $81.51 \pm 4.33 \%$, while the proportion of cells in the $\mathrm{S}$ phase significantly decreased from $8.75 \pm 2.13$ to $3.61 \pm 2.05 \%$ (both $\mathrm{P}<0.05$; Fig. 5E).

\section{Discussion}

S100A11 is widely expressed in multiple tissues and exists as a noncovalenthomodimerwith an anti-parallel conformation. The binding of $\mathrm{Ca}^{2+}$ triggers conformational changes of S100A11, which facilitates its interaction with target proteins. S100A11 has been reported to be overexpressed in numerous different types of human cancer, including renal cell carcinoma, lung adenocarcinoma and papillary thyroid carcinoma (23-26). Our previous study revealed that S100A11 serves an important role in the development and prognosis of pancreatic cancer (15). However, the exact role of S100A11 in the pancreatic cancer remained unknown. In order to investigate the molecular mechanism underlying the tumor-promoting role of S100A11 in the occurrence and development of pancreatic cancer, an in vitro model of S100A11 overexpression in pancreatic cancer PANC-1 cells was successfully constructed. High expression levels of S100A11 are able to regulate cell cycle progression and the proliferation of pancreatic cancer cells $(12,14)$. The results of the present study revealed that the overexpression of S100A11 significantly promoted human pancreatic cancer PANC-1 cell proliferation, reduced the percentage of early apoptotic cells, and enhanced the G1/S phase transition of pancreatic cancer cells. These results were consistent with previous studies of ovarian and lung tumors $(27,28)$.

The PI3K/AKT signaling pathway serves an important function in the development of pancreatic cancer (19-21). However, it remains unclear whether the PI3K/AKT signaling pathway participated in the action of S100A11 in pancreatic cancer. Firstly, immunohistochemistry was used to analyze the correlation between S100A11 and p-AKT, which suggested a positive correlation between of S100A11 and p-AKT expression in the pancreatic cancer tissues of 30 cases. Furthermore, using an in vitro cell model, it was demonstrated that the overexpression of S100A11 significantly increased AKT mRNA expression, and total AKT and p-AKT protein expression. In order to further investigate the association between S100A11 and the PI3K/AKT signaling pathway in pancreatic cancer, PANC-1 cells were treated with LY294002, a specific inhibitor of PI3K. Through the specific inhibition of PI3 catalytic activity, LY294002 treatment leads to the dephosphorylation of downstream AKT at T308 and S473 residues (29). It has been acknowledged that the dephosphorylation of AKT inhibits cell growth and induces cell apoptosis. In the current study, LY294002 was employed to inhibit the phosphorylation of AKT, thereby specifically inhibiting the PI3K/AKT pathway. The results from the present study revealed that LY294002 treatment significantly inhibited cell proliferation, and promoted cell apoptosis and cell cycle arrest in the G0/G1 phase in PANC-1 cells. These results were supported by previous studies in human keratinocyte cells, which reported that the PI3K/AKT signaling pathway is involved in S100A11-triggered signal transduction $(22,30)$. Of note, it was demonstrated that treatment with LY294002 significantly attenuated AKT mRNA expression and the protein expression of p-AKT. However, the mRNA of S100A11, and protein expression levels of S100A11 and total AKT were not affected. Through AKT is rapidly activated through protein phosphorylation, certain studies have also documented that increased levels of total AKT is a hallmark of tumor cells (31-33). The mRNA and protein levels of AKT are regulated by complex mechanisms, and involve numerous signaling molecules, including glycogen synthase kinase (GSK)-3 $\beta$, Twist, microRNA (miR)-185 and miR-203 (34-37). Therefore, we hypothesized that LY294002 may regulate the activities of these molecules, particularly GSK-3 $\beta$, which is a well-known AKT substrate, thus exerting an influence on AKT mRNA expression, but not on the total AKT protein expression. However, the potential mechanism underlying this effect remains elusive and warrants further investigation.

To conclude, the results of the current study demonstrated that S100A11 promoted human pancreatic cancer PANC-1 cell proliferation through the PI3K/AKT signaling pathway. LY294002 was able to significantly inhibit the proliferation of PANC-1 cells, and induce apoptosis through the PI3K/AKT pathway without affecting the expression of S100A11 mRNA and protein levels, suggesting that existence of S100A11 

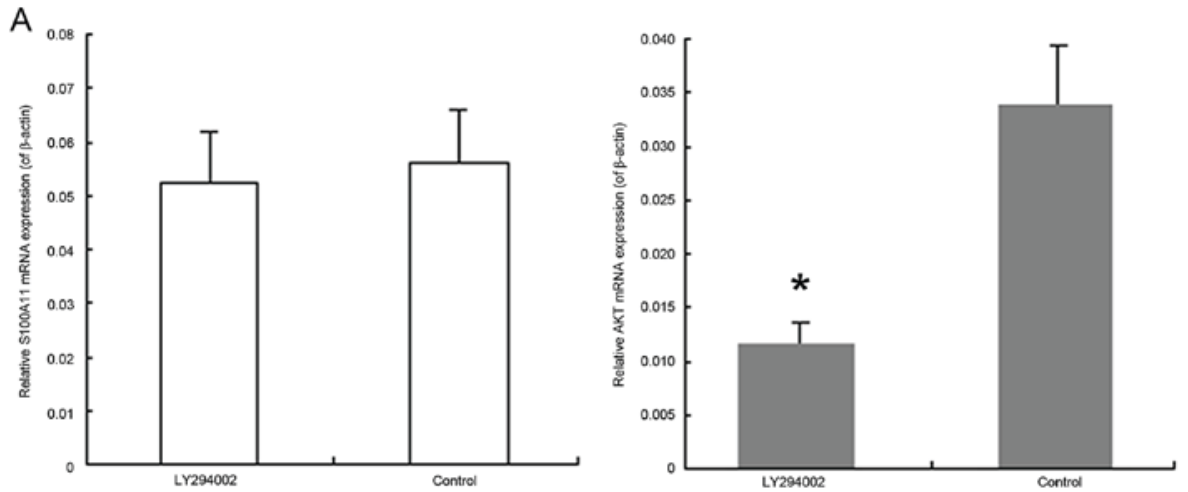

B
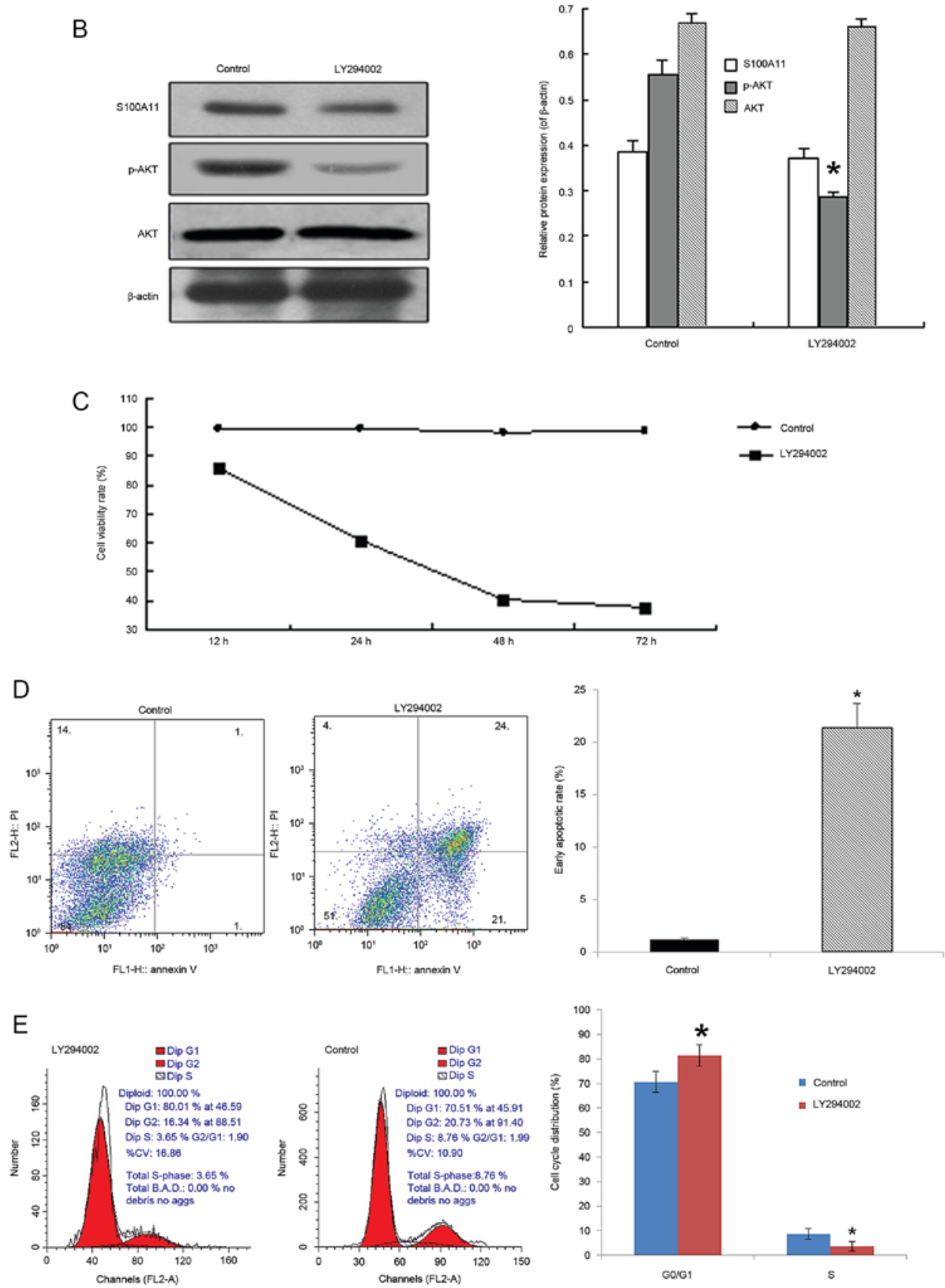

Figure 5. Effects of PI3K inhibitor LY294002 on cell proliferation, apoptosis, cell cycle distribution, mRNA expression of S100A11 and AKT, and protein expression of S100A11, p-AKT and total AKT in PANC-1 cells. PANC-1 cells were treated with $50 \mu \mathrm{mol} / 1 \mathrm{LY} 294002$ for $48 \mathrm{~h}$. (A) mRNA expression of S100A11 was determined by semi-quantitative reverse transcription-polymerase chain reaction analysis. $\beta$-actin was used as an internal control. (B) Protein expression of S100A11, p-AKT and total AKT were determined by western blot analysis. $\beta$-actin was used as an internal control. (C) Cell proliferation was determined using a cell counting kit-8 assay. (D) Cell apoptosis was determined by flow cytometry analysis using Annexin V/PI staining. (E) Cell cycle distribution was determined by flow cytometry analysis using PI staining. Data were presented as the mean \pm standard error of mean following three independent experiments. " $\mathrm{P}<0.05$ vs. control. p, phosphorylated; AKT, AKT serine/threonine kinase; PI, propidium iodide. 
activity upstream of the PI3K/Akt signaling pathway. However, the specific mechanism regarding the role of S100A11 in the development of pancreatic cancer requires further studies. S100A11 may serve as a potentially effective target in pancreatic cancer therapy.

\section{Acknowledgements}

The present study was supported by grants from the Natural Youth Science Foundation of China (grant no. 81502055), The Health Project of Jiangsu Province (grant no. H201624), The Natural Science Foundation of Jiangsu Province (grant no. BK20161286) and The Social Development Foundation of Nantong City (grant nos. MS22016056, MS22015062, HS2014072 and MS22015044).

\section{References}

1. Ryan DP, Hong TS and Bardeesy N: Pancreatic adenocarcinoma. N Engl J Med 371: 1039-1049, 2014.

2. Zell JA, Rhee JM, Ziogas A, Lipkin SM and Anton-Culver H: Race, socioeconomic status, treatment and survival time among pancreatic cancer cases in California. Cancer Epidemiol Biomarkers Prev 16: 546-552, 2007.

3. Lau MK, Davila JA and Shaib YH: Incidence and survival of pancreatic head and body and tail cancers: A population-based study in the United States. Pancreas 39: 458-462, 2010.

4. Quaresma M, Coleman MP and Rachet B: 40-year trends in an index of survival for all cancers combined and survival adjusted for age and sex for each cancer in England and Wales, 1971-2011: A population-based study. Lancet 385: 1206-1218, 2015.

5. Lin QJ, Yang F, Jin C and Fu DL: Current status and progress of pancreatic cancer in China. World J Gastroenterol 21: 7988-8003, 2015.

6. Yu J, Blackford AL, Dal Molin M, Wolfgang CL and Goggins M: Time to progression of pancreatic ductal adenocarcinoma from low-to-high tumour stages. Gut 64: 1783-1789, 2015.

7. Polyak K, Lee MH, Erdjument-Bromage H, Koff A, Roberts JM, Tempst $\mathrm{P}$ and Massagué J: Cloning of p27Kip1, a cyclin-dependent kinase inhibitor and a potential mediator of extracellular antimitogenic signals. Cell 78: 59-66, 1994

8. Yang F, Di Y, Li J, Wang XY, Yao L, Hao SJ, Jiang YJ, Jin C and Fu DL: Accuracy of routine multidetector computed tomography to identify arterial variants in patients scheduled for pancreaticoduodenectomy. World J Gastroenterol 21: 969-976, 2015.

9. Arumugam T and Logsdon CD: S100P: A novel therapeutic target for cancer. Amino Acids 41: 893-899, 2011.

10. Hung KW, Chang YM and Yu C: Resonance assignments of $\mathrm{Ca}^{2+}$-bound human S100A11. Biomol NMR Assign 7: 211-214, 2013.

11. Sakaguchi M and Huh NH: S100A11, a dual growth regulator of epidermal keratinocytes. Amino Acids 41: 797-807, 2011.

12. Ji YF, Huang H, Jiang F, Ni RZ and Xiao MB: S100 family signaling network and related proteins in pancreatic cancer (Review). Int J Mol Med 33: 769-776, 2014.

13. Ohuchida K, Mizumoto K, Ohhashi S, Yamaguchi H, Konomi H, Nagai E, Yamaguchi K, Tsuneyoshi M and Tanaka M: S100A11, A putative tumor suppressor gene, is overexpressed in pancreatic carcinogenesis. Clin Cancer Res 12: 5417-5422, 2006.

14. Chen H, Xu C, Jin Q and Liu Z: S100 protein family in human cancer. Am J Cancer Res 4: 89-115, 2014.

15. Xiao MB, Jiang F, Ni WK, Chen BY, Lu CH, Li XY and Ni RZ: High expression of S100A11 in pancreatic adenocarcinoma is an unfavorable prognostic marker. Med Oncol 29: 1886-1891, 2012.

16. Donato R, Cannon BR, Sorci G, Riuzzi F, Hsu K, Weber DJ and Geczy CL: Functions of S100 proteins. Curr Mol Med 13: 24-57, 2013.

17. Yamamoto S, Tomita Y, Hoshida Y, Morooka T, Nagano $\mathrm{H}$, Dono K, Umeshita K, Sakon M, Ishikawa O, Ohigashi H, et al: Prognostic significance of activated Akt expression in pancreatic ductal adenocarcinoma. Clin Cancer Res 10: 2846-2850, 2004

18. Jiang H, Fan D, Zhou G, Li X and Deng H: Phosphatidylinositol 3-kinase inhibitor (LY294002) induces apoptosis of human nasopharyngeal carcinoma in vitro and in vivo. J Exp Clin Cancer Res 29: 34, 2010.
19. Asano T, Yao Y, Zhu J, Li D, Abbruzzese JL and Reddy SA: The PI 3-kinase/Akt signaling pathway is activated due to aberrant Pten expression and targets transcription factors NF-kappaB and c-Myc in pancreatic cancer cells. Oncogene 23: 8571-8580, 2004.

20. Chadha KS, Khoury T, Yu J, Black JD, Gibbs JF, Kuvshinoff BW, Tan D, Brattain MG and Javle MM: Activated Akt and Erk expression and survival after surgery in pancreatic carcinoma. Ann Surg Oncol 13: 933-939, 2006.

21. Roy SK, Srivastava RK and Shankar S: Inhibition of PI3K/AKT and MAPK/ERK pathways causes activation of FOXO transcription factor, leading to cell cycle arrest and apoptosis in pancreatic cancer. J Mol Signal 5: 10, 2010.

22. Sakaguchi M, Sonegawa H, Murata H, Kitazoe M, Futami J, Kataoka K, Yamada H and Huh NH: S100A11, an dual mediator for growth regulation of human keratinocytes. Mol Biol Cell 19: 78-85, 2008

23. Gabril M, Girgis H, Scorilas A, Rotondo F, Wala S, Bjarnason GA, Ding Q, Evans A, Tawedrous E, Pasic M, et al: S100A11 is a potential prognostic marker for clear cell renal cell carcinoma. Clin Exp Metastasis 33: 63-71, 2016.

24. Woo T, Okudela K, Mitsui H, Tajiri M, Rino Y, Ohashi K and Masuda M: Up-regulation of S100A11 in lung adenocarcinoma-its potential relationship with cancer progression. PLoS One 10: e0142642, 2015.

25. Anania MC, Miranda C, Vizioli MG, Mazzoni M, Cleris L, Pagliardini S, Manenti G, Borrello MG, Pierotti MA and Greco A: S100A11 overexpression contributes to the malignant phenotype of papillary thyroid carcinoma. J Clin Endocrinol Metab 98: E1591-E1600, 2013.

26. Wang G, Wang X, Wang S, Song H, Sun H, Yuan W, Cao B, Bai J and Fu S: Colorectal cancer progression correlates with upregulation of S100A11 expression in tumor tissues. Int J Colorectal Dis 23: 675-682, 2008.

27. Liu Y, Han X and Gao B: Knockdown of S100A11 expression suppresses ovarian cancer cell growth and invasion. Exp Ther Med 9: 1460-1464, 2015.

28. Hao J, Wang K, Yue Y, Tian T, Xu A, Hao J, Xiao X and He D: Selective expression of S100A11 in lung cancer and its role in regulating proliferation of adenocarcinomas cells. Mol Cell Biochem 359: 323-332, 2012.

29. Gong C, Liao H, Wang J, Lin Y, Qi J, Qin L, Tian LQ and Guo FJ: LY294002 induces G0/G1 cell cycle arrest and apoptosis of cancer stem-like cells from human osteosarcoma via down-regulation of PI3K activity. Asian Pac J Cancer Prev 13: 3103-3107, 2012.

30. Foertsch F, Teichmann N, Kob R, Hentschel J, Laubscher U and Melle C: S100A11 is involved in the regulation of the stability of cell cycle regulator p21 (CIP1/WAF1) in human keratinocyte HaCaT cells. FEBS J 280: 3840-3853, 2013

31. Fresno Vara JA, Casado E, de Castro J, Cejas P, Belda-Iniesta C and Gonzalez-Baron M: PI3K/Akt signalling pathway and cancer. Cancer Treat Rev 30: 193-204, 2004.

32. Vivanco I and Sawyers CL: The phosphatidylinositol 3-kinase AKT pathway in human cancer. Nat Rev Cancer 2: 489-501, 2002.

33. Le Page C, Koumakpayi IH, Alam-Fahmy M, Mes-Masson AM and Saad F: Expression and localisation of Akt-1, Akt-2 and Akt-3 correlate with clinical outcome of prostate cancer patients. Br J Cancer 94: 1906-1912, 2006.

34. Nemoto T, Kanai T, Yanagita T, Satoh S, Maruta T, Yoshikawa N, Kobayashi $\mathrm{H}$ and Wada A: Regulation of Akt mRNA and protein levels by glycogen synthase kinase-3beta in adrenal chromaffin cells: Effects of LiCl and SB216763. Eur J Pharmacol 586: 82-89, 2008.

35. Tsitoura E, Wells AU, Karagiannis K, Lasithiotaki I, Vasarmidi E, Bibaki E, Koutoulaki C, Sato H, Spandidos DA, Siafakas NM, et al: MiR-185/AKT and miR-29a/Collagen 1a pathways are activated in IPF BAL cells. Oncotarget 7: 74569-74581, 2016.

36. Li J, Chen Y, Zhao J, Kong F and Zhang Y: miR-203 reverses chemoresistance in p53-mutated colon cancer cells through downregulation of Akt2 expression. Cancer Lett 304: 52-59, 2011

37. Cheng GZ, Chan J, Wang Q, Zhang W, Sun CD and Wang LH: Twist transcriptionally up-regulates AKT2 in breast cancer cells leading to increased migration, invasion and resistance to paclitaxel. Cancer Res 67: 1979-1987, 2007. International (CC BY-NC-ND 4.0) License. 